\title{
Stabilization and $L_{2}$-gain analysis for a class of cascade switched nonlinear systems: An average dwell-time method
}

\author{
Ben Niu ${ }^{\mathrm{a}, *}$, Jun Zhao ${ }^{\mathrm{a}, \mathrm{b}}$ \\ ${ }^{a}$ Key Laboratory of Integrated Automation of Process Industry, Ministry of Education, Northeastern University, Shenyang, 110819, PR China \\ ${ }^{\mathrm{b}}$ Research School of Information Sciences and Engineering, The Australian National University, Canberra ACT 0200, Australia
}

\section{A R T I C L E I N F O}

\section{Article history:}

Received 13 July 2010

Accepted 17 May 2011

\section{Keywords:}

Switched cascade systems

Average dwell-time

Asymptotic stability

Weighted $L_{2}$-gain

\begin{abstract}
A B S T R A C T
This paper is concerned with the problem of stabilization and $L_{2}$-gain analysis for a class of cascade switched nonlinear systems by using the average dwell-time method. First, when all subsystems are stabilizable, we design a state feedback controller and an average dwell-time scheme, which guarantee that the corresponding closed-loop system is globally asymptotically stable and has a weighted $L_{2}$-gain. Then, we extend the result to the case where not all subsystems are stabilizable, under the condition that the activation time ratio between stabilizable subsystems and unstabilizable ones is not less than a specified constant, we also derive sufficient conditions for the stabilization and weighted $L_{2}$-gain property. Finally, an example is given to illustrate the effectiveness of our results.
\end{abstract}

(c) 2011 Elsevier Ltd. All rights reserved.

\section{Introduction}

In recent years, switched systems have gained considerable interest in both theoretical research and practical application [1-6]. This is motivated by the need for systematic and formal methods to investigate such systems. Stability analysis is one of the fundamental problems in the study of switched systems. In this aspect, the Lyapunov approach and its variants still play an important role. Stability under arbitrary switchings is guaranteed by the existence of a Common Lyapunov function $[7,8]$. However, Common Lyapunov functions are often too conservative in order to assess stability for switched systems. This problem may be avoided by using a different strategy, such as multiple Lyapunov functions [9-11] or average dwell-time constraints [12,13].

Nonlinear cascade systems are composed of two parts, where control input only enters one part, state change of the other one can be "controlled" by the interconnections between these two parts. With the development of geometric theory for nonlinear systems, it is known that many nonlinear systems with reduced relative degree can be transformed into nonlinear cascade systems under certain conditions. Therefore, the study of nonlinear cascade systems has been a hot topic in the control area [14,15]. For such systems, considerable attention has been paid to robust control problem [16], invariance control problem [17], etc. Especially, the $L_{2}$-gain disturbance attenuation problem has been concerned in [18-21], and it should be mentioned that the problem is very difficult because these results usually require solving Hamilton-Jacobi inequalities.

On the other hand, for switched systems, because of the complicated behavior caused by the interaction between the continuous dynamics and discrete switching, the stabilization and $L_{2}$-gain analysis problem of switched cascade systems is more difficult to study. Only a few results have appeared in the literature and most of the results are about the systems that are linear or nonlinear containing linear parts [22-24]. The importance of the study of stabilization and $L_{2}$-gain analysis problem for switched cascade systems arises from the extensive applications in actual control systems; see e.g., $[25,26]$.

\footnotetext{
* Corresponding author. Tel.: +86 13664148817; fax: +86 05394489339.

E-mail addresses: niubengj@163.com, niubenjk@gmail.com (B. Niu), zhaojun@mail.neu.edu.cn (J. Zhao).
} 
However, to the best of the authors' knowledge, few results on the topic for cascade switched nonlinear systems with stabilizable and unstabilizable subsystems have been reported by now.

In this paper, we discuss the stabilization and $L_{2}$-gain analysis problem for a class of cascade switched nonlinear systems by using the average dwell-time method. Each subsystem under consideration is composed of two cascade-connected parts: a nonlinear one which contain disturbance input and a linearizable and controllable one. Sufficient conditions for the stabilization and weighted $L_{2}$-gain of the switched system are derived. State feedback controllers for subsystems and certain average dwell-time based switching laws are designed to achieve global asymptotic stability of the resulting closedloop system with the weighted $L_{2}$-gain property.

Unlike the previous works on the stabilization and $L_{2}$-gain analysis problem for switched systems, this paper owns three features. First, we concern the cascade switched nonlinear systems where the disturbance input appears in all system equations. Second, after considering the case where all subsystems are stabilizable, unstabilizable subsystems are permitted to exist to reduce conservativeness. Third, with the help of the generalized inverse of matrices, the dimensions of the control input and the state variables in the controllable part of the switched system are allowed to be different, which covers more general cases.

\section{Problem formulation and preliminaries}

In this paper, we consider a class of cascade switched nonlinear systems of the form:

$$
\left\{\begin{array}{l}
\dot{x}_{1}(t)=f_{1, \sigma(t)}\left(x_{1}(t), x_{2}(t)\right)+c_{\sigma(t)}\left(x_{1}(t), x_{2}(t)\right) \omega(t), \\
\dot{x}_{2}(t)=f_{2, \sigma(t)}\left(x_{1}(t), x_{2}(t)\right)+p_{\sigma(t)}\left(x_{1}(t), x_{2}(t)\right) u_{\sigma(t)}(t)+b_{\sigma(t)}\left(x_{1}(t), x_{2}(t)\right) \omega(t), \\
y(t)=h_{\sigma(t)}\left(x_{1}(t), x_{2}(t)\right)+d_{\sigma(t)}\left(x_{1}(t), x_{2}(t)\right) \omega(t),
\end{array}\right.
$$

where $x_{1}(t) \in R^{n-d}, x_{2}(t) \in R^{d}$ are the states, $\omega(t) \in L_{2}[0, \infty)$ is the external disturbance input, and $y(t) \in R^{p}$ is the controlled output. $\sigma(t):[0, \infty) \rightarrow M=\{1, \ldots, N\}$ is the switching signal, which is a right continuous piecewise constant function of time and will be determined later. $u_{i}(t) \in R^{m}$ is the control input, and $f_{1, i}(\cdot, \cdot), f_{2, i}(\cdot, \cdot), c_{i}(\cdot, \cdot), p_{i}(\cdot, \cdot), b_{i}(\cdot, \cdot)$, $h_{i}(\cdot, \cdot), d_{i}(\cdot, \cdot)$ are smooth real functions with $f_{1, i}(0,0)=0, f_{2, i}(0,0)=0, h_{i}(0,0)=0$, for $\forall\left(x_{1}^{T}(t), x_{2}^{T}(t)\right)^{T} \in R^{n}, i=1$, $\ldots, N$.

Corresponding to the switching signal $\sigma(t)$, we have the switching sequence $\sum=\left\{\left(x_{1}^{T}\left(t_{0}\right), x_{2}^{T}\left(t_{0}\right)\right)^{T} ;\left(i_{0}, t_{0}\right),\left(i_{1}, t_{1}\right), \ldots\right.$, $\left.\left(i_{k}, t_{k}\right), \ldots, \mid i_{k} \in M, k=0,1, \ldots\right\}$, which means that the $i_{k}$ th subsystems is active when $t \in\left[t_{k}, t_{k+1}\right)$. In addition, we assume that the state of the switched system (1) does not jump at the switching instants.

Remark 1. The switched system (1) is more general than many models of the existing results, see e.g., [17-20,22-26]. The advantage of the system under consideration is that all parts of each subsystem are nonlinear. Furthermore, the disturbance input $\omega(t)$ is allowed to enter all system equations, which is more realistic.

From a practical consideration, the disturbance gains $d_{i}(\cdot, \cdot)$ in the output of $(1)$ are usually bounded. Hence, we shall make the following assumption.

Assumption 1. There exists a positive constant $\gamma_{d}$ such that

$$
d_{i}^{T}\left(x_{1}(t), x_{2}(t)\right) d_{i}\left(x_{1}(t), x_{2}(t)\right) \leq \gamma_{d}^{2} I, \quad \forall\left(x_{1}^{T}(t), x_{2}^{T}(t)\right)^{T} \in R^{n}, i \in M .
$$

The problem to be addressed in this paper is stated as follows: Given a positive scalar $\gamma>0$, for each $i$, design a state feedback controller

$$
u_{i}(t)=u_{i}\left(x_{1}(t), x_{2}(t)\right)
$$

with $u_{i}(0,0)=0$ and a switching law $\sigma=\sigma(t)$ such that the resulting closed-loop system of (1) satisfies the following conditions:

(i) Internal stability. The system

$$
\left\{\begin{array}{l}
\dot{x}_{1}(t)=f_{1, \sigma(t)}\left(x_{1}(t), x_{2}(t)\right) \\
\dot{x}_{2}(t)=f_{2, \sigma(t)}\left(x_{1}(t), x_{2}(t)\right)+p_{\sigma(t)}\left(x_{1}(t), x_{2}(t)\right) u_{\sigma(t)}\left(x_{1}(t), x_{2}(t)\right)
\end{array}\right.
$$

is asymptotically stable.

(ii) Weighted $L_{2}$-gain. That is, the following inequality holds for some real-valued function $\beta\left(x_{1}(t), x_{2}(t)\right)$ with $\beta(0,0)=0$

$$
\int_{0}^{\infty} \mathrm{e}^{-\lambda \tau} y^{T}(\tau) y(\tau) \mathrm{d} \tau \leq \gamma^{2} \int_{0}^{\infty} \omega^{T}(\tau) \omega(\tau) \mathrm{d} \tau+\beta\left(x_{1}(0), x_{2}(0)\right) .
$$

We focus on switching laws with some average dwell-time given by the following definition. 
Definition 1 ([12]). For any switching signal $\sigma(t)$ and any $t>\tau>0$, let $N_{\sigma}(\tau, t)$ denote the number of switchings of $\sigma(t)$ on the interval $(\tau, t)$. If

$$
N_{\sigma}(\tau, t) \leq N_{0}+\frac{t-\tau}{\tau_{a}}
$$

holds for $N_{0} \geqslant 0, \tau_{a}>0$. The constant $\tau_{a}$ is called average dwell-time and $N_{0}$ is the chatter bound. As commonly used in the literature, we choose $N_{0}=0$.

Definition 2. For the switched system (1), suppose that $V_{i}(x)$ is the corresponding Lyapunov function for the $i$ th subsystem, then $V(t)$ is called a piecewise Lyapunov function candidate if it can be written as $V(t)=V_{\sigma(t)}(x)$, where $V_{\sigma(t)}(x)$ is switched among $V_{i}(x)$ in accordance with the piecewise constant switching signal $\sigma(t)$.

Remark 2. The standard $L_{2}$-gain, which has been commonly adopted for non-switched systems, cannot be achieved in general for switched systems with switchings satisfying a certain average dwell-time and thus weight $L_{2}$-gain with the weighted term $\mathrm{e}^{-\lambda \tau}$ is used instead in the literature [13,22].

\section{Main results}

In this section, we solve the stabilization and $L_{2}$-gain analysis problem for the switched systems (1). We first address the problem when all subsystems are stabilizable. Then, we generalize the result to the case where not all subsystems are stabilizable.

First of all, we write the functions $f_{1, i}\left(x_{1}, x_{2}\right), c_{i}\left(x_{1}, x_{2}\right), h_{i}\left(x_{1}, x_{2}\right)$ in the form:

$$
\begin{aligned}
& f_{1, i}\left(x_{1}, x_{2}\right)=f_{1, i}\left(x_{1}, 0\right)+\tilde{f}_{1, i}\left(x_{1}, x_{2}\right) x_{2} \\
& c_{i}\left(x_{1}, x_{2}\right)=c_{i}\left(x_{1}, 0\right)+\tilde{c}_{i}\left(x_{1}, x_{2}\right) x_{2} \quad i=1, \ldots, N . \\
& h_{i}\left(x_{1}, x_{2}\right)=h_{i}\left(x_{1}, 0\right)+\tilde{h}_{i}\left(x_{1}, x_{2}\right) x_{2} .
\end{aligned}
$$

For the case where all subsystems are stabilizable, the following result shows that the stabilization and $L_{2}$-gain analysis problem of the switched system (1) are solvable when the subsystems are switched by an average dwell-time scheme.

Theorem 1. Given any constant $\gamma>\gamma_{d}$, suppose that the switched system (1) satisfies the following conditions:

(1) There exist radially unbounded positive definite differentiable functions $V_{i}\left(x_{1}\right), i=1, \ldots, N$, constants $\gamma_{1}>0, \lambda_{0}>0$, and $\mu \geq 1$, such that

$$
\begin{aligned}
& \frac{\partial V_{i}}{\partial x_{1}} f_{1, i}\left(x_{1}, 0\right)+\frac{1}{4 \gamma_{1}^{2}} \frac{\partial V_{i}}{\partial x_{1}} c_{i}\left(x_{1}, 0\right) c_{i}^{T}\left(x_{1}, 0\right) \frac{\partial V_{i}^{T}}{\partial x_{1}}+h_{i}^{T}\left(x_{1}, 0\right) h_{i}\left(x_{1}, 0\right)+\lambda_{0} V_{i} \leq 0, \\
& V_{i}\left(x_{1}\right) \leq \mu V_{j}\left(x_{1}\right), \quad i, j=1, \ldots, N,
\end{aligned}
$$

and

$$
\alpha_{1}^{*}\left(\left\|x_{1}\right\|\right) \leq V_{i}\left(x_{1}\right) \leq \alpha_{2}^{*}\left(\left\|x_{1}\right\|\right),
$$

where $\alpha_{1}^{*}$ and $\alpha_{2}^{*}$ are two class $K_{\infty}$ functions.

(2) The matrices $p_{i}\left(x_{1}, x_{2}\right)$ have the $M-P$ generalized inverses $p_{i}^{+}\left(x_{1}, x_{2}\right)$, such that

$$
p_{i}\left(x_{1}, x_{2}\right) p_{i}^{+}\left(x_{1}, x_{2}\right) F_{i}\left(x_{1}, x_{2}\right)=F_{i}\left(x_{1}, x_{2}\right)
$$

holds for $\forall\left(x_{1}^{T}, x_{2}^{T}\right)^{T} \in R^{n}, i=1, \ldots, N$. Where $F_{i}\left(x_{1}, x_{2}\right)=f_{2, i}\left(x_{1}, x_{2}\right)+\tilde{f}_{1, i}^{T}\left(x_{1}, x_{2}\right) \frac{\partial V_{i}^{T}}{\partial x_{1}}+2 \tilde{h}_{i}^{T}\left(x_{1}, x_{2}\right) h_{i}\left(x_{1}, 0\right)+\frac{1}{4 \gamma_{4}^{2}}\left(b_{i}\right.$ $\left.\left(x_{1}, x_{2}\right)\right)^{2} x_{2}+\frac{1}{4 \gamma_{2}^{2}} \tilde{c}_{i}^{T}\left(x_{1}, x_{2}\right) \frac{\partial V_{i}^{T}}{\partial x_{1}} \frac{\partial V_{i}}{\partial x_{1}} \tilde{c}_{i}\left(x_{1}, x_{2}\right) x_{2}+\tilde{h}_{i}^{T}\left(x_{1}, x_{2}\right) \tilde{h}_{i}\left(x_{1}, x_{2}\right) x_{2}+\frac{\lambda_{0}}{2} x_{2}$, and $\gamma_{2}, \gamma_{4}$ are some properly chosen constants.

Then, the switched system (1) is globally asymptotically stabilizable when $\omega \equiv 0$ and has a weighted $L_{2}$-gain level $\gamma$ with the state feedback controller

$$
u_{i}\left(x_{1}, x_{2}\right)=-p_{i}^{+}\left(x_{1}, x_{2}\right) F_{i}\left(x_{1}, x_{2}\right)
$$

and under an arbitrary switching law which satisfies the average dwell-time

$$
\tau_{a} \geq \tau_{a}^{*}=\frac{\log \mu}{\lambda},
$$

where $\lambda$ can be chosen in the open set $\left(0, \lambda_{0}\right)$.

Proof. See the Appendix. 
Remark 3. (6) can be considered as a type of Hamilton-Jacobi inequalities which are often adopted in the literature on switched systems, see e.g., [13,22]. For switched systems, (7)-(8) are assumptions that are commonly made when the average dwell-time method is applied. (9) is used to handle a general case where $p_{i}\left(x_{1}, x_{2}\right)$ are allowed to be rectangular matrices. When $p_{i}\left(x_{1}, x_{2}\right)$ are square matrices, which is often addressed in the literature $[16,18,26]$, (9) is automatically satisfied.

Now, we consider the case that not all subsystems are stabilizable, in addition to the average dwell-time scheme, we employ a switching condition so as for the switched system (1) to achieve a finite weighted $L_{2}$-gain, where the total activation time ratio between stabilizable and unstabilizable subsystems is required to be smaller than a specified constant.

Let $M_{p}$ denote a proper nonempty subset of $M, \tilde{M}_{p}$ denote a complement of $M_{p}$ with respect to $M$. If $i \in M_{p}$, then the $i$ th subsystem is stabilizable, otherwise, if $i \in \tilde{M}_{p}$, then the $i$ th subsystem is unstabilizable.

For any switching signal and any $t>\tau \geq 0$, we let $T^{+}(\tau, t)$ (resp., $T^{-}(\tau, t)$ ) denote the total activation time of unstabilizable subsystems (resp., stabilizable subsystems) during the interval $\left[\tau, t\right.$ ). Denote $\lambda^{+}=\max _{i \in \tilde{M}_{p}}\left\{-\lambda_{i} \mid \lambda_{i}<0\right\}$, $\lambda^{-}=\min _{i \in M_{p}}\left\{\lambda_{i} \mid \lambda_{i}>0\right\}$, and choose an arbitrary $\lambda^{*} \in\left(0, \lambda^{-}\right)$. Motivated by the idea in [13], we employ the following switching strategy:

(S) Determine the switching signal $\sigma(t)$ such that the following inequality holds for any given initial time $t_{0}$

$$
\inf _{t \geqslant t_{0}} \frac{T^{-}\left(t_{0}, t\right)}{T^{+}\left(t_{0}, t\right)} \geq \frac{\lambda^{+}+\lambda^{*}}{\lambda^{-}-\lambda^{*}}
$$

From the switching strategy (S) and the discussion of the previous section, we obtain the following result immediately.

Theorem 2. Given any constant $\gamma>\gamma_{d}$, and positive constants $\lambda_{i}$ for $i \in M_{p}$, negative constants $\lambda_{i}$ for $i \in \tilde{M}_{p}$. Suppose that the switched system (1) satisfies the following conditions:

(1) There exist radially unbounded positive definite differentiable functions $V_{i}\left(x_{1}\right), i=1, \ldots, N$, constants $\gamma_{1}>0$, and $\mu \geq 1$, such that

$$
\begin{aligned}
& \frac{\partial V_{i}}{\partial x_{1}} f_{1, i}\left(x_{1}, 0\right)+\frac{1}{4 \gamma_{1}^{2}} \frac{\partial V_{i}}{\partial x_{1}} c_{i}\left(x_{1}, 0\right) c_{i}^{T}\left(x_{1}, 0\right) \frac{\partial V_{i}^{T}}{\partial x_{1}}+h_{i}^{T}\left(x_{1}, 0\right) h_{i}\left(x_{1}, 0\right)+\lambda_{i} V_{i} \leq 0, \\
& V_{i}\left(x_{1}\right) \leq \mu V_{j}\left(x_{1}\right), \quad i, j=1, \ldots, N,
\end{aligned}
$$

and

$$
\alpha_{1}^{*}\left(\left\|x_{1}\right\|\right) \leq V_{i}\left(x_{1}\right) \leq \alpha_{2}^{*}\left(\left\|x_{1}\right\|\right),
$$

where $\alpha_{1}^{*}$ and $\alpha_{2}^{*}$ are two class $K_{\infty}$ functions.

(2) The matrices $p_{i}\left(x_{1}, x_{2}\right)$ have the $M-P$ generalized inverses $p_{i}^{+}\left(x_{1}, x_{2}\right)$, such that

$$
p_{i}\left(x_{1}, x_{2}\right) p_{i}^{+}\left(x_{1}, x_{2}\right) F_{i}\left(x_{1}, x_{2}\right)=F_{i}\left(x_{1}, x_{2}\right)
$$

holds for $\forall\left(x_{1}^{T}, x_{2}^{T}\right)^{T} \in R^{n}, i=1, \ldots, N$. Where $F_{i}\left(x_{1}, x_{2}\right)=f_{2, i}\left(x_{1}, x_{2}\right)+\tilde{f}_{1, i}^{T}\left(x_{1}, x_{2}\right) \frac{\partial V_{i}^{T}}{\partial x_{1}}+2 \tilde{h}_{i}^{T}\left(x_{1}, x_{2}\right) h_{i}\left(x_{1}, 0\right)+\frac{1}{4 \gamma_{4}^{2}}\left(b_{i}\right.$ $\left.\left(x_{1}, x_{2}\right)\right)^{2} x_{2}+\frac{1}{4 \gamma_{2}^{2}} \tilde{c}_{i}^{T}\left(x_{1}, x_{2}\right) \frac{\partial V_{i}^{T}}{\partial x_{1}} \frac{\partial V_{i}}{\partial x_{1}} \tilde{c}_{i}\left(x_{1}, x_{2}\right) x_{2}+\tilde{h}_{i}^{T}\left(x_{1}, x_{2}\right) \tilde{h}_{i}\left(x_{1}, x_{2}\right) x_{2}+\frac{\lambda_{i}}{2} x_{2}$, and $\gamma_{2}, \gamma_{4}$ are some properly chosen constants.

Then, the switched system (1) is globally asymptotically stabilizable when $\omega \equiv 0$ and has the weighted $L_{2}$-gain property with the state feedback controller

$$
u_{i}\left(x_{1}, x_{2}\right)=-p_{i}^{+}\left(x_{1}, x_{2}\right) F_{i}\left(x_{1}, x_{2}\right)
$$

and under an arbitrary switching signal $\sigma(t)$ with average dwell-time (4) and switching condition (S) satisfying

$$
\tau_{a} \geq \tau_{a}^{*}=\frac{\ln \mu}{\lambda},
$$

where $\lambda$ can be chosen in the open set $\left(0, \lambda^{*}\right)$.

Proof. See the Appendix.

Remark 4. From the proof of Theorem 2, when $\mu=1$, we known that a common Lyapunov function can be found for the closed-loop system (1). From (38) we have $\tau_{0}^{*}=0$, which implies that the global asymptotic stabilization problem of the switched system (1) is solvable with an arbitrary switching law under the switching condition (S). From (42), we know that the closed-loop system (1) has the standard $L_{2}$-gain for the trivial case of $\mu=1$.

\section{Example}

In this section, we provide a numerical example to demonstrate the proposed results. In what follows, for the sake of simplicity, we shall omit the dependence on $t$ for all functions wherever no confusion will be caused. 


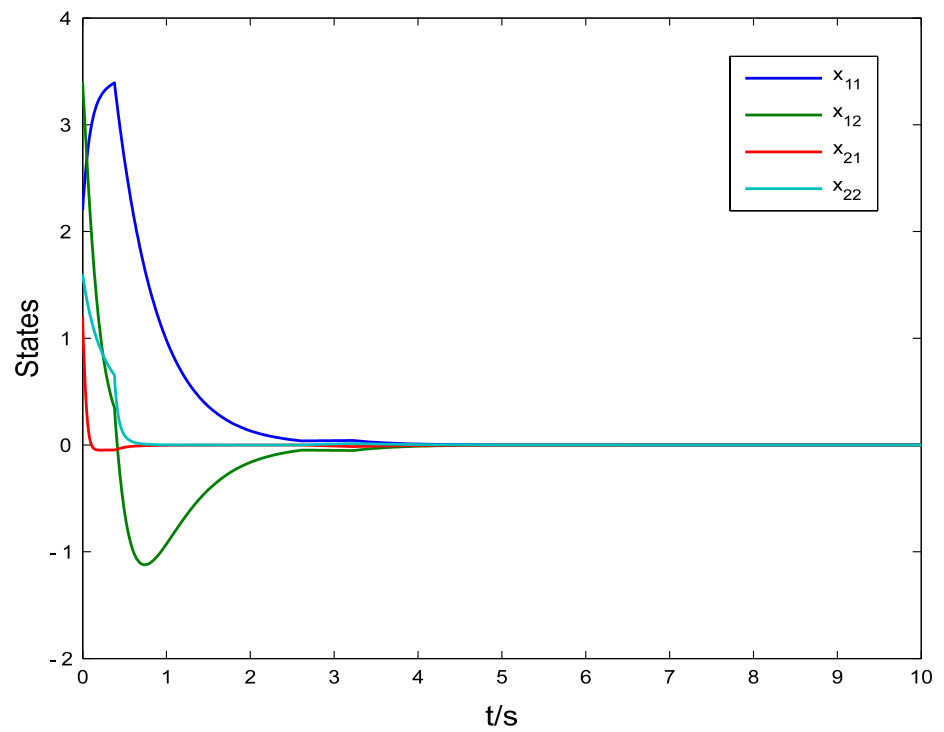

Fig. 1. The state responses of the switched system.

Consider the switched system (1) with $M=\{1,2\}, n=4, d=2, x_{1}=\left(x_{11}, x_{12}\right)^{T}, x_{2}=\left(x_{21}, x_{22}\right)^{T}$, and

$$
\begin{aligned}
& f_{1,1}\left(x_{1}, x_{2}\right)=\left[\begin{array}{c}
-2 x_{11}+0.25 x_{21}^{2} \\
-3 x_{11}-4.4 x_{12}+0.25 x_{22}^{2}
\end{array}\right], \quad c_{1}\left(x_{1}, x_{2}\right)=\left[\begin{array}{c}
0 \\
x_{22}
\end{array}\right], \quad p_{1}\left(x_{1}, x_{2}\right)=\left[\begin{array}{l}
10 \\
01
\end{array}\right], \\
& f_{2,1}\left(x_{1}, x_{2}\right)=\left[\begin{array}{c}
-x_{21}+0.25 x_{11} \\
-x_{22}+0.2 x_{12}
\end{array}\right], \quad b_{1}\left(x_{1}, x_{2}\right)=\left[\begin{array}{l}
x_{11} \\
x_{12}
\end{array}\right], \quad d_{1}\left(x_{1}, x_{2}\right)=\left[\begin{array}{c}
0.7 \cos \left(x_{11}\right) \\
0.7 \cos \left(x_{11}\right)
\end{array}\right], \\
& h_{1}\left(x_{1}, x_{2}\right)=\left[\begin{array}{l}
0.5 x_{11} \\
0.5 x_{12}
\end{array}\right], \quad f_{1,2}\left(x_{1}, x_{2}\right)=\left[\begin{array}{c}
0.1 x_{11}+x_{12}^{2} \\
0.2 x_{12}-2 x_{11} x_{12}
\end{array}\right], \quad c_{2}\left(x_{1}, x_{2}\right)=\left[\begin{array}{c}
x_{21} \\
0
\end{array}\right], \\
& p_{2}\left(x_{1}, x_{2}\right)=\left[\begin{array}{l}
10 \\
01
\end{array}\right], \quad f_{2,2}\left(x_{1}, x_{2}\right)=\left[\begin{array}{l}
x_{21}\left(1+x_{22}^{2}\right)+0.2 x_{11} \\
x_{22}\left(1+x_{21}^{2}\right)+0.2 x_{12}
\end{array}\right], \quad b_{2}\left(x_{1}, x_{2}\right)=\left[\begin{array}{c}
x_{21} \\
x_{22}
\end{array}\right], \\
& d_{2}\left(x_{1}, x_{2}\right)=\left[\begin{array}{l}
0.6 \sin \left(x_{21}\right) \\
0.6 \sin \left(x_{21}\right)
\end{array}\right], \quad h_{2}\left(x_{1}, x_{2}\right)=\left[\begin{array}{l}
\sqrt{0.1} x_{11}+x_{21} \\
\sqrt{0.1} x_{12}+x_{22}
\end{array}\right] .
\end{aligned}
$$

It is easy to check that the first subsystem is stabilizable and the second one is unstabilizable. Let $\gamma=3, \gamma_{d}=1$, on the base of $\gamma_{1}^{2}+\gamma_{2}^{2}+\gamma_{4}^{2}=\frac{\gamma_{3}^{2}\left(\gamma^{2}-\gamma_{3}^{2}-\gamma_{d}^{2}\right)}{\gamma_{3}^{2}+\gamma_{d}^{2}}$, we can choose $\gamma_{1}=\sqrt{2,} \gamma_{2}=1, \gamma_{3}=\sqrt{2}, \gamma_{4}=1$. We also make $\lambda_{1}=0.8, \lambda_{2}=-0.5$, and then $\lambda^{-}=0.8, \lambda^{+}=0.5$. Choose $V_{1}\left(x_{1}\right)=1.5 x_{11}^{2}+0.8 x_{11} x_{12}+1.5 x_{12}^{2}, V_{2}\left(x_{1}\right)=2 x_{11}^{2}+x_{12}^{2}$, from (12) we can get

$$
\begin{aligned}
& \frac{\partial V_{1}}{\partial x_{1}} f_{1,1}\left(x_{1}, 0\right)+\frac{1}{4 \gamma_{1}^{2}} \frac{\partial V_{1}}{\partial x_{1}} c_{1}\left(x_{1}, 0\right) c_{1}^{T}\left(x_{1}, 0\right) \frac{\partial V_{1}^{T}}{\partial x_{1}}+h_{1}^{T}\left(x_{1}, 0\right) h_{1}\left(x_{1}, 0\right)+\lambda_{1} V_{1}\left(x_{1}\right) \\
& =-6.95 x_{11}^{2}-13.48 x_{11} x_{12}-11.75 x_{12}^{2} \leq 0, \\
& \frac{\partial V_{1}}{\partial x_{1}} f_{1,2}\left(x_{1}, 0\right)+\frac{1}{4 \gamma_{1}^{2}} \frac{\partial V_{1}}{\partial x_{1}} c_{2}\left(x_{1}, 0\right) c_{2}^{T}\left(x_{1}, 0\right) \frac{\partial V_{1}^{T}}{\partial x_{1}}+h_{2}^{T}\left(x_{1}, 0\right) h_{2}\left(x_{1}, 0\right)+\lambda_{2} V_{2}\left(x_{1}\right)=-0.5 x_{11}^{2} \leq 0 .
\end{aligned}
$$

Let $\mu=1.6, \lambda^{*}=0.5$ and $\lambda=0.496$, we obtain $\tau_{a}^{*}=0.95$ and $T^{-}(0, t) / T^{+}(0, t) \geqslant\left(\lambda^{+}+\lambda^{*}\right) /\left(\lambda^{-}-\lambda^{*}\right)=3.33$. In addition, the state feedback controller (16) can be constructed as:

$$
\begin{aligned}
& u_{1}=-\left[\begin{array}{l}
0.4 x_{21}+0.75 x_{11} x_{21}+0.2 x_{12} x_{21}+0.25 x_{11}^{2} x_{21}+0.25 x_{11} x_{12} x_{22}+0.25 x_{11} \\
0.4 x_{22}+0.75 x_{12} x_{24}+0.2 x_{11} x_{22}+2.5 x_{12}^{2} x_{22}+0.25 x_{11} x_{12} x_{21}+0.6 x_{11} x_{12} x_{22}+1.6 x_{11}^{2} x_{22}+0.2 x_{12}
\end{array}\right], \\
& u_{2}=-\left[\begin{array}{l}
(0.2+2 \sqrt{0.1}) x_{11}+2.25 x_{21}+0.25 x_{21}^{3}+1.25 x_{21} x_{22}^{2}+4 x_{11}^{2} x_{21} \\
(0.2+2 \sqrt{0.1}) x_{12}+2.25 x_{22}+0.25 x_{22}^{3}+1.25 x_{21}^{2} x_{22}
\end{array}\right] .
\end{aligned}
$$

By using the switching scheme provided by Theorem 2, the closed-loop system of (1) is globally asymptotically stable when $\omega \equiv 0$ and has the weighted $L_{2}$-gain property. The simulation results are depicted in Figs. 1 and 2. 


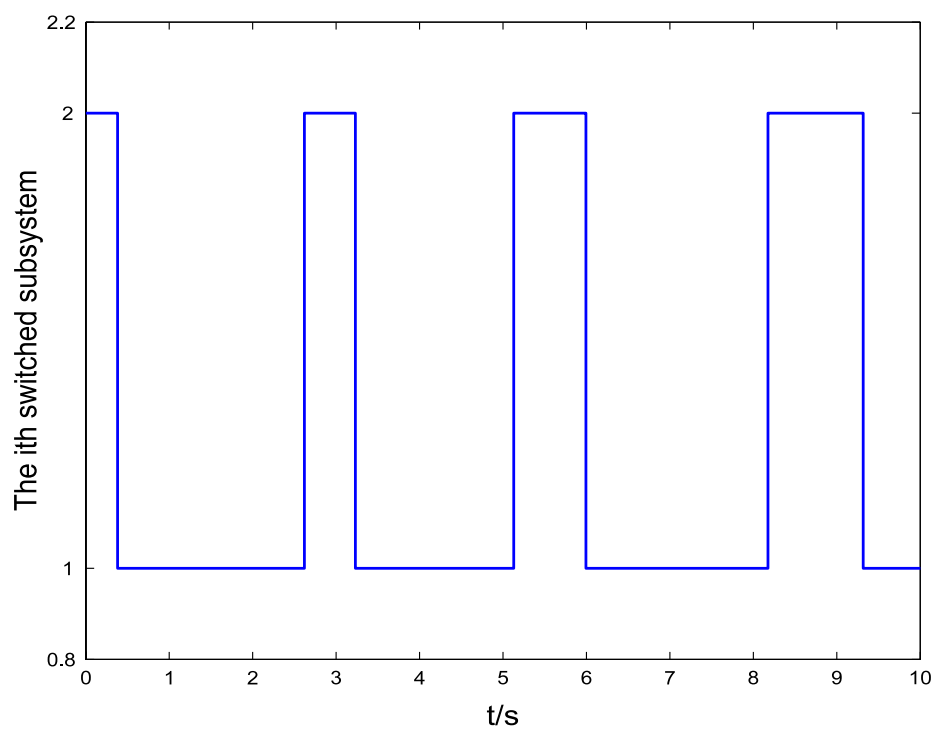

Fig. 2. The switching signals for the switched system.

\section{Conclusions}

This paper has focused on the stabilization and $L_{2}$-gain analysis problem for a class of cascade switched nonlinear systems by using the average dwell-time method. Sufficient conditions are derived for the switched system to be globally asymptotically stabilizable with the weighted $L_{2}$-gain property via switching and associated state feedback controllers. Our results allow unstabilizable subsystems to exist and the theory of the generalized inverse is used to release the conservative hypothesis that the dimensions of the control input and the state variables in the controllable part of each subsystem are identical. The feasibility of the proposed results has been illustrated through a numerical example.

\section{Acknowledgments}

The authors are grateful to the associate editor and the anonymous reviewers for their valuable comments and suggestions for further improving the quality and presentation of this paper. This work was supported by the National Natural Science Foundation of China under Grants 60874024 and 90816028, and the SRFDP under Grant 200801450019.

\section{Appendix}

Proof of Theorem 1. For arbitrary $t>0$, denote $t_{0} \leq t_{1} \leq t_{2} \leq \cdots \leq t_{k} \cdots \leq t_{N_{\sigma}\left(t_{0}, t\right)}$ as the switching instants of $\sigma(t)$ over the interval $\left(t_{0}, t\right)$ and $N_{\sigma}\left(t_{0}, t\right)$ as the number of switchings of $\sigma(t)$ in the interval $\left(t_{0}, t\right)$. Without loss of generality, we further assume that the initial time $t_{0}=0$.

From Definition 2, we choose the following piecewise Lyapunov function candidate

$$
V^{*}(t)=V_{\sigma(t)}^{*}(x)=V_{\sigma(t)}\left(x_{1}\right)+\frac{1}{2} x_{2}^{T} x_{2},
$$

where $V_{i}\left(x_{1}\right)$ satisfy (6)-(8).

In view of (5), when $\sigma(t)=i$, differentiating $V^{*}$ along the trajectory of the switched system (1) gives rise to

$$
\begin{aligned}
\dot{V}^{*}= & \frac{\partial V_{i}}{\partial x_{1}}\left[f_{1, i}\left(x_{1}, x_{2}\right)+c_{i}\left(x_{1}, x_{2}\right) \omega\right]+x_{2}^{T}\left[f_{2, i}\left(x_{1}, x_{2}\right)+p_{i}\left(x_{1}, x_{2}\right) u_{i}+b_{i}\left(x_{1}, x_{2}\right) \omega\right] \\
= & \frac{\partial V_{i}}{\partial x_{1}}\left[f_{1, i}\left(x_{1}, 0\right)+\tilde{f}_{1, i}\left(x_{1}, x_{2}\right) x_{2}+c_{i}\left(x_{1}, 0\right) \omega+\tilde{c}_{i}\left(x_{1}, x_{2}\right) x_{2} \omega\right] \\
& +x_{2}^{T}\left[f_{2, i}\left(x_{1}, x_{2}\right)+p_{i}\left(x_{1}, x_{2}\right) u_{i}+b_{i}\left(x_{1}, x_{2}\right) \omega\right] .
\end{aligned}
$$

Base on the formula $a b \leq \frac{1}{4 \gamma^{2}} a^{2}+\gamma^{2} b^{2}, \gamma \neq 0$, we can get

$$
\dot{V}^{*} \leq \frac{\partial V_{i}}{\partial x_{1}} f_{1, i}\left(x_{1}, 0\right)+\frac{1}{4 \gamma_{1}^{2}}\left(\frac{\partial V_{i}}{\partial x_{1}} c_{i}\left(x_{1}, 0\right)\right)^{2}+x_{2}^{T}\left[f_{2, i}\left(x_{1}, x_{2}\right)+p_{i}\left(x_{1}, x_{2}\right) u_{i}+\tilde{f}_{1, i}^{T}\left(x_{1}, x_{2}\right) \frac{\partial V_{i}^{T}}{\partial x_{1}}\right.
$$




$$
\left.+b_{i}\left(x_{1}, x_{2}\right) \omega+\frac{1}{4 \gamma_{2}^{2}}\left(\frac{\partial V_{i}}{\partial x_{1}} \tilde{c}_{i}\left(x_{1}, x_{2}\right)\right)^{2} x_{2}\right]+\gamma_{1}^{2} \omega^{T} \omega+\gamma_{2}^{2} \omega^{T} \omega .
$$

Using Assumption 1, we obtain

$$
\begin{aligned}
y^{T} y-\gamma^{2} \omega^{T} \omega & =h_{i}^{T}\left(x_{1}, x_{2}\right) h_{i}\left(x_{1}, x_{2}\right)+2 h_{i}\left(x_{1}, x_{2}\right) d_{i}\left(x_{1}, x_{2}\right) \omega+d_{i}^{T}\left(x_{1}, x_{2}\right) d_{i}\left(x_{1}, x_{2}\right) \omega^{T} \omega-\gamma^{2} \omega^{T} \omega \\
& \leq h_{i}^{T}\left(x_{1}, x_{2}\right) h_{i}\left(x_{1}, x_{2}\right)+\frac{\gamma_{d}^{2}}{\gamma_{3}^{2}} h_{i}^{T}\left(x_{1}, x_{2}\right) h_{i}\left(x_{1}, x_{2}\right)-\left(\gamma^{2}-\gamma_{3}^{2}-\gamma_{d}^{2}\right) \omega^{T} \omega \\
& =\left(1+\frac{\gamma_{d}^{2}}{\gamma_{3}^{2}}\right) h_{i}^{T}\left(x_{1}, x_{2}\right) h_{i}\left(x_{1}, x_{2}\right)-\left(\gamma^{2}-\gamma_{3}^{2}-\gamma_{d}^{2}\right) \omega^{T} \omega,
\end{aligned}
$$

where $\gamma_{3}>0$ is a constant satisfying $\gamma^{2}-\gamma_{3}^{2}-\gamma_{d}^{2}>0$.

Combining the previous two inequalities gives

$$
\begin{aligned}
\dot{V}^{*}+\frac{\gamma_{3}^{2}}{\gamma_{3}^{2}+\gamma_{d}^{3}}\left(y^{T} y-\gamma^{2} \omega^{T} \omega\right) \leq & \frac{\partial V_{i}}{\partial x_{1}} f_{1, i}\left(x_{1}, 0\right)+\frac{1}{4 \gamma_{1}^{2}}\left(\frac{\partial V_{i}}{\partial x_{1}} c_{i}\left(x_{1}, 0\right)\right)^{2}+h_{i}^{T}\left(x_{1}, 0\right) h_{i}\left(x_{1}, 0\right) \\
& +x_{2}\left[f_{2, i}\left(x_{1}, x_{2}\right)+\tilde{f}_{1, i}^{T}\left(x_{1}, x_{2}\right) \frac{\partial V_{i}^{T}}{\partial x_{1}}+p_{i}\left(x_{1}, x_{2}\right) u_{i}\right. \\
& +\tilde{h}_{i}^{T}\left(x_{1}, x_{2}\right) \tilde{h}_{i}\left(x_{1}, x_{2}\right) x_{2}+2 \tilde{h}_{i}^{T}\left(x_{1}, x_{2}\right) h_{i}\left(x_{1}, 0\right) \\
& \left.+\frac{1}{4 \gamma_{4}^{2}}\left(b_{i}\left(x_{1}, x_{2}\right)\right)^{2} x_{2}+\frac{1}{4 \gamma_{2}^{2}}\left(\frac{\partial V_{i}}{\partial x_{1}} \tilde{c}_{i}\left(x_{1}, x_{2}\right)\right)^{2} x_{2}\right] \\
& +\left(\gamma_{1}^{2}+\gamma_{2}^{2}+\gamma_{4}^{2}\right) \omega^{T} \omega-\frac{\gamma_{3}^{2}\left(\gamma^{2}-\gamma_{3}^{2}-\gamma_{d}^{2}\right)}{\gamma_{3}^{2}+\gamma_{d}^{2}} \omega^{T} \omega .
\end{aligned}
$$

Let $\gamma_{2}^{2}=\frac{\gamma_{3}^{2}\left(\gamma^{2}-\gamma_{3}^{2}-\gamma_{d}^{2}\right)}{\gamma_{3}^{2}+\gamma_{d}^{2}}-\gamma_{1}^{2}-\gamma_{4}^{2}$, and based on the controller (10), it holds that

$$
\begin{aligned}
\dot{V}^{*}+\frac{\gamma_{3}^{2}}{\gamma_{3}^{2}+\gamma_{d}^{2}}\left(y^{T} y-\gamma^{2} \omega^{T} \omega\right) & \leq \frac{\partial V_{i}}{\partial x_{1}} f_{1, i}\left(x_{1}, 0\right)+\frac{1}{4 \gamma_{1}^{2}}\left(\frac{\partial V_{i}}{\partial x_{1}} c_{i}\left(x_{1}, 0\right)\right)^{2}+h_{i}^{T}\left(x_{1}, 0\right) h_{i}\left(x_{1}, 0\right)-\frac{1}{2} \lambda_{0} x_{2}^{T} x_{2} \\
& \leq-\lambda_{0} V_{i}\left(x_{1}\right)-\frac{1}{2} \lambda_{0} x_{2}^{T} x_{2} \\
& =-\lambda_{0} V^{*} .
\end{aligned}
$$

When $\omega=0$, from the above inequality, we obtain

$$
\dot{V}^{*} \leq-\lambda_{0} V^{*} \text {. }
$$

According to (7), it holds that

$$
V_{i}^{*}(t) \leq \mu V_{j}^{*}(t), \quad i, j=1, \ldots, N .
$$

Then, from (23) and (24), we have

$$
V^{*}(t) \leq \mu^{N_{\sigma}(0, t)} \mathrm{e}^{-\lambda_{0} t} V^{*}(0)=\mathrm{e}^{N_{\sigma}(0, t) \ln u-\lambda_{0} t} V^{*}(0) .
$$

Here, we assume $V^{*}\left(t_{k}\right)$ is the value of the Lyapunov function $V^{*}(t)$ at the switching instant $t_{k}, k=0,1, \ldots, N_{\sigma}(0, t) . V^{*}$ $\left(t_{N_{\sigma}(0, t)}^{-}\right)$is the value of the Lyapunov function $V^{*}(t)$ at the former instant of the switching instant $t_{N_{\sigma}(0, t)}$.

Furthermore, in view of $N_{\sigma}(0, \tau) \leq \tau / \tau_{a}^{*}$ for $\forall \tau>0,(11)$ implies that

$$
N_{\sigma}(0, \tau) \ln \mu \leq \lambda \tau \text {. }
$$

Thus,

$$
V^{*}(t) \leq \mathrm{e}^{-\left(\lambda_{0}-\lambda\right) t} V^{*}(0) .
$$

From (8) and (18), we know that there exist two class $K_{\infty}$ functions $\alpha_{1}$ and $\alpha_{2}$, such that

$$
\alpha_{1}(\|x\|) \leq V^{*}(t) \leq \alpha_{2}(\|x\|) .
$$

In fact, we can choose $\alpha_{1}(x)=\alpha_{1}^{*}\left(\left\|x_{1}\right\|\right)+\frac{1}{2}\left\|x_{2}\right\|^{2}, \alpha_{1}(x)=\alpha_{2}^{*}\left(\left\|x_{1}\right\|\right)+\frac{1}{2}\left\|x_{2}\right\|^{2}$. 
Combining (27)-(28) gives

$$
\|x(t)\| \leq \alpha_{1}^{-1}\left(\mathrm{e}^{-\left(\lambda_{0}-\lambda\right) t} \alpha_{2}(\|x(0)\|)\right),
$$

which implies global asymptotic stability of the closed-loop system (1) when $\omega(t) \equiv 0$.

Integrating both sides of (22) and from (24), we have

$$
\begin{aligned}
V^{*}(t) \leq & V^{*}\left(t_{N_{\sigma}(0, t)}\right) \mathrm{e}^{-\lambda_{0}\left(t-t_{N_{\sigma}(0, t)}\right)}-\int_{t_{N_{\sigma}(0, t)}}^{t} \mathrm{e}^{-\lambda_{0}(t-\tau)} \Gamma(\tau) \mathrm{d} \tau \\
\leq & \mu\left[V^{*}\left(t_{N_{\sigma}(0, t)-1}\right) \mathrm{e}^{-\lambda_{0}\left(t_{N_{\sigma}(0, t)}-t_{N_{\sigma}(0, t)-1}\right)}-\int_{t_{N_{\sigma}(0, t)-1}}^{t_{N_{\sigma}(0, t)}} \mathrm{e}^{-\lambda_{0}\left(t_{N_{\sigma}(0, t)}-\tau\right)} \Gamma(\tau) \mathrm{d} \tau\right] \mathrm{e}^{-\lambda_{0}\left(t-t_{N_{\sigma}(0, t)}\right)} \\
& -\int_{t_{N_{\sigma}(0, t)}^{t}}^{t} \mathrm{e}^{-\lambda_{0}(t-\tau)} \Gamma(\tau) \mathrm{d} \tau \leq \cdots \\
\leq & \mu^{N_{\sigma}(0, t)} \mathrm{e}^{-\lambda_{0} t} V^{*}(0)-\mu^{N_{\sigma}(0, t)} \int_{0}^{t_{1}} \mathrm{e}^{-\lambda_{0}(t-\tau)} \Gamma(\tau) \mathrm{d} \tau-\mu^{N_{\sigma}(0, t)-1} \int_{t_{1}}^{t_{2}} \mathrm{e}^{-\lambda_{0}(t-\tau)} \Gamma(\tau) \mathrm{d} \tau \\
& -\cdots-\mu^{0} \int_{t_{N_{\sigma}(0, t)}}^{t} \mathrm{e}^{-\lambda_{0}(t-\tau)} \Gamma(\tau) \mathrm{d} \tau \\
= & \mathrm{e}^{-\lambda_{0} t+N_{\sigma}(0, t) \ln \mu} V^{*}(0)-\int_{0}^{t} \mathrm{e}^{-\lambda_{0}(t-\tau)+N_{\sigma}(\tau, t) \ln \mu} \Gamma(\tau) \mathrm{d} \tau,
\end{aligned}
$$

where $\Gamma(\tau)=\frac{\gamma_{3}^{2}}{\gamma_{3}^{2}+\gamma_{d}^{2}}\left[y^{T}(\tau) y(\tau)-\gamma^{2} \omega^{T}(\tau) \omega(\tau)\right]$.

Multiplying both sides of the above inequality by $\mathrm{e}^{-N_{\sigma}(0, t) \ln \mu}$ and considering (26), we can get

$$
\int_{0}^{t} \mathrm{e}^{-\lambda_{0}(t-\tau)-\lambda \tau} y^{T}(\tau) y(\tau) \mathrm{d} \tau \leq \frac{\gamma_{3}^{2}+\gamma_{d}^{2}}{\gamma_{3}^{2}} \mathrm{e}^{-\lambda_{0} t} V^{*}(0)+\gamma^{2} \int_{0}^{t} \mathrm{e}^{-\lambda_{0}(t-\tau)} \omega^{T}(\tau) \omega(\tau) \mathrm{d} \tau .
$$

Integrating both sides of the foregoing inequality from $t=0$ to $\infty$ and rearranging the double-integral area, we have

$$
\int_{0}^{\infty} \mathrm{e}^{-\lambda \tau} y^{T}(\tau) y(\tau) \mathrm{d} \tau \leq \frac{\gamma_{3}^{2}+\gamma_{d}^{2}}{\gamma_{3}^{2}} V^{*}(0)+\gamma^{2} \int_{0}^{\infty} \omega^{T}(\tau) \omega(\tau) \mathrm{d} \tau .
$$

From (3), we know that the closed-loop system (1) has the weighted $L_{2}$-gain level $\gamma$.

Proof of Theorem 2. Similar to the proof of Theorem 1, we define the following Lyapunov function candidate

$$
V^{*}(t)=V_{\sigma(t)}^{*}(x)=V_{\sigma}\left(x_{1}\right)+\frac{1}{2} x_{2}^{T} x_{2},
$$

where $V_{i}\left(x_{1}\right)$ satisfy (12)-(14).

Then, when the ith switched subsystem is activate, based on (16), we can get

$$
\dot{V}_{i}^{*}+\frac{\gamma_{3}^{2}}{\gamma_{3}^{2}+\gamma_{d}^{2}}\left(y^{T} y-\gamma^{2} \omega^{T} \omega\right) \leq-\lambda_{i} V_{i}^{*} .
$$

When $\omega \equiv 0$, from the above inequality, we obtain

$$
\dot{V}_{i}^{*} \leq-\lambda_{i} V_{i}^{*} \text {. }
$$

From (34), we know that for any $t \in\left[t_{k}, t_{k+1}\right)\left(0 \leq k \leq N_{\sigma}(0, t)\right)$, the piecewise Lyapunov function candidate (32) satisfies

$$
V^{*}(t)=V_{\sigma(t)}^{*}(t) \leq \begin{cases}\mathrm{e}^{-\lambda^{-}\left(t-t_{k}\right)} V_{\sigma\left(t_{k}\right)}^{*}\left(t_{k}\right), & i \in M_{p}, \\ \mathrm{e}^{\lambda^{+}\left(t-t_{k}\right)} V_{\sigma\left(t_{k}\right)}^{*}\left(t_{k}\right), & i \in \tilde{M}_{p} .\end{cases}
$$

From (13), $V_{\sigma\left(t_{k}\right)}^{*}\left(t_{k}\right) \leq \mu V_{\sigma\left(t_{k}^{-}\right)}^{*}\left(t_{k}^{-}\right)$is true at the switching point $t_{k}$. Therefore, we obtain by induction that

$$
\begin{aligned}
V^{*}(t) & \leq \mathrm{e}^{\lambda^{+} T^{+}\left(t_{k}, t\right)-\lambda^{-} T^{-}\left(t_{k}, t\right)} V_{\sigma\left(t_{k}\right)}^{*}\left(t_{k}\right) \\
& \leq \mu \mathrm{e}^{\lambda^{+} T^{+}\left(t_{k}, t\right)-\lambda^{-} T^{-}\left(t_{k}, t\right)} V_{\sigma\left(t_{k}^{-}\right)}^{*}\left(t_{k}^{-}\right)
\end{aligned}
$$




$$
\begin{aligned}
& \leq \mu \mathrm{e}^{\lambda^{+} T^{+}\left(t_{k-1}, t\right)-\lambda^{-} T^{-}\left(t_{k-1}, t\right)} V_{\sigma\left(t_{k-1}\right)}^{*}\left(t_{k-1}\right) \\
& \leq \cdots \leq \mu^{N_{\sigma}(0, t)} \mathrm{e}^{\lambda^{+} T^{+}(0, t)-\lambda^{-} T^{-}(0, t)} V_{\sigma(0)}^{*}(0) \\
& =\mathrm{e}^{\lambda^{+} T^{+}(0, t)-\lambda^{-} T^{-}(0, t)+N_{\sigma}(0, t) \ln \mu} V_{\sigma(0)}^{*}(0) .
\end{aligned}
$$

From (14) and (32), we know that there exist two class $K_{\infty}$ functions $\alpha_{1}$ and $\alpha_{2}$, such that

$$
\alpha_{1}(\|x\|) \leq V^{*}(t) \leq \alpha_{2}(\|x\|) .
$$

In fact, we can choose $\alpha_{1}(x)=\alpha_{1}^{*}\left(\left\|x_{1}\right\|\right)+\frac{1}{2}\left\|x_{2}\right\|^{2}, \alpha_{1}(x)=\alpha_{2}^{*}\left(\left\|x_{1}\right\|\right)+\frac{1}{2}\left\|x_{2}\right\|^{2}$.

Combining (35)-(37) gives

$$
\|x(t)\| \leq \alpha_{1}^{-1}\left(\mathrm{e}^{\lambda^{+} T^{+}(0, t)-\lambda^{-} T^{-}(0, t)+N_{\sigma}(0, t) \ln \mu} \alpha_{2}(\|x(0)\|)\right) .
$$

When $\mu=1$, which is a trivial case, we obtain from the switching strategy (S) that

$$
\|x(t)\| \leq \alpha_{1}^{-1}\left(\mathrm{e}^{-\lambda^{*} t} \alpha_{2}(\|x(0)\|)\right),
$$

which means that the switched system (1) is globally asymptotically stabilizable under the switching condition (S) without considering the average dwell-time (17).

For the nontrivial case of $\mu>1$, it follows from the average dwell-time (17) and the switching condition (S) that

$$
\lambda^{+} T^{+}(0, t)-\lambda^{-} T^{-}(0, t)+N_{\sigma}(0, t) \ln \mu \leq-\left(\lambda^{*}-\lambda\right) t .
$$

Then, $\|x(t)\| \leq \alpha_{1}^{-1}\left(\mathrm{e}^{-\left(\lambda^{*}-\lambda\right) t} \alpha_{2}(\|x(0)\|)\right)$, which implies global asymptotic stability of the closed-loop system (1) when $\omega(t) \equiv 0$.

Next, we show that the closed-loop system (1) has the weighted $L_{2}$-gain property. It can be easily seen from (33) that for any $t \in\left[t_{k}, t_{k+1}\right)\left(0 \leq k \leq N_{\sigma}(0, t)\right)$, the piecewise Lyapunov function candidate (32) satisfies

$$
V^{*}(t) \leq\left\{\begin{array}{l}
\mathrm{e}^{-\lambda^{-}\left(t-t_{k}\right)} V_{\sigma\left(t_{k}\right)}^{*}\left(t_{k}\right)-\int_{t_{k}}^{t} \mathrm{e}^{-\lambda^{-}(t-\tau)} \Gamma(\tau) \mathrm{d} \tau, \quad i \in M_{p}, \\
\mathrm{e}^{\lambda^{+}\left(t-t_{k}\right)} V_{\sigma\left(t_{k}\right)}^{*}\left(t_{k}\right)-\int_{t_{k}}^{t^{t}} \mathrm{e}^{\lambda^{+}(t-\tau)} \Gamma(\tau) \mathrm{d} \tau, \quad i \in \tilde{M}_{p},
\end{array}\right.
$$

where $\Gamma(\tau)=\frac{\gamma_{3}^{2}}{\gamma_{3}^{2}+\gamma_{d}^{2}}\left[y^{T}(\tau) y(\tau)-\gamma^{2} \omega^{T}(\tau) \omega(\tau)\right]$.

From (13), $V_{\sigma\left(t_{k}\right)}^{*}\left(t_{k}\right) \leq \mu V_{\sigma\left(t_{k}^{-}\right)}^{*}\left(t_{k}^{-}\right)$is true at the switching point $t_{k}$. Therefore, we obtain by induction that

$$
\begin{aligned}
V^{*}(t) \leq & \mathrm{e}^{\lambda^{+} T^{+}\left(t_{k}, t\right)-\lambda^{-} T^{-}\left(t_{k}, t\right)} V_{\sigma\left(t_{k}\right)}^{*}\left(t_{k}\right)-\int_{t_{k}}^{t} \mathrm{e}^{\lambda^{+} T^{+}(\tau, t)-\lambda^{-} T^{-}(\tau, t)} \Gamma(\tau) \mathrm{d} \tau \\
\leq & \mathrm{e}^{\lambda^{+} T^{+}\left(t_{k}, t\right)-\lambda^{-} T^{-}\left(t_{k}, t\right)} \mu V_{\sigma\left(t_{k}^{-}\right)}^{*}\left(t_{k}^{-}\right)-\int_{t_{k}}^{t} \mathrm{e}^{\lambda^{+} T^{+}(\tau, t)-\lambda^{-} T^{-}(\tau, t)} \Gamma(\tau) \mathrm{d} \tau \\
\leq & \mathrm{e}^{\lambda^{+} T^{+}\left(t_{k-1}, t\right)-\lambda^{-} T^{-}\left(t_{k-1}, t\right)} \mu V_{\sigma\left(t_{k-1}\right)}^{*}\left(t_{k-1}\right)-\int_{t_{k}}^{t} \mathrm{e}^{\lambda^{+} T^{+}(\tau, t)-\lambda^{-} T^{-}(\tau, t)} \Gamma(\tau) \mathrm{d} \tau \\
& -\mathrm{e}^{\lambda^{+} T^{+}\left(t_{k}, t\right)-\lambda^{-} T^{-}\left(t_{k}, t\right)} \mu \int_{t_{k-1}}^{t_{k}} \mathrm{e}^{\lambda^{+} T^{+}(\tau, t)-\lambda^{-} T^{-}(\tau, t)} \Gamma(\tau) \mathrm{d} \tau \leq \cdots \\
\leq & \mathrm{e}^{\lambda^{+} T^{+}(0, t)-\lambda^{-} T^{-}(0, t)} \mu^{N_{\sigma}(0, t)} V_{\sigma(0)}^{*}(0)-\int_{0}^{t} \mu^{N_{\sigma}(\tau, t)} \mathrm{e}^{\lambda^{+} T^{+}(\tau, t)-\lambda^{-} T^{-}(\tau, t)} \Gamma(\tau) \mathrm{d} \tau \\
= & \mathrm{e}^{\lambda^{+} T^{+}(0, t)-\lambda^{-} T^{-}(0, t)+N_{\sigma}(0, t) \ln \mu} V_{\sigma(0)}^{*}(0)-\int_{0}^{t} \mathrm{e}^{\lambda^{+} T^{+}(\tau, t)-\lambda^{-} T^{-}(\tau, t)+N_{\sigma}(\tau, t) \ln \mu} \Gamma(\tau) \mathrm{d} \tau .
\end{aligned}
$$

For the trivial case of $\mu=1,(40)$ and (S) give that

$$
\int_{0}^{t} \mathrm{e}^{-\lambda^{-}(t-\tau)} y^{T}(\tau) y(\tau) \mathrm{d} \tau \leq \frac{\gamma_{3}^{2}+\gamma_{d}^{2}}{\gamma_{3}^{2}} \mathrm{e}^{-\lambda^{*} t} V_{\sigma(0)}^{*}(0)+\gamma^{2} \int_{0}^{t} \mathrm{e}^{-\lambda^{*}(t-\tau)} \omega^{T}(\tau) \omega(\tau) \mathrm{d} \tau .
$$

Integrating both sides of this inequality from $t=0$ to $\infty$ and rearranging the double-integral area, we obtain

$$
\begin{aligned}
\int_{0}^{\infty} y^{T}(\tau) y(\tau) \mathrm{d} \tau & \leq \frac{\lambda^{-}\left(\gamma_{3}^{2}+\gamma_{d}^{2}\right)}{\lambda^{*} \gamma_{3}^{2}} V_{\sigma(0)}^{*}(0)+\frac{\lambda^{-} \gamma^{2}}{\lambda^{*}} \int_{0}^{\infty} \omega^{T}(\tau) \omega(\tau) \mathrm{d} \tau \\
& =\frac{\lambda^{-}\left(\gamma_{3}^{2}+\gamma_{d}^{2}\right)}{\lambda^{*} \gamma_{3}^{2}} V_{\sigma(0)}^{*}(0)+\gamma_{0}^{2} \int_{0}^{\infty} \omega^{T}(\tau) \omega(\tau) \mathrm{d} \tau,
\end{aligned}
$$


where $\gamma_{0}=\sqrt{\frac{\lambda^{-}}{\lambda^{*}}} \gamma$, which means that the closed-loop system (1) has finite $L_{2}$-gain under switching law (S) without considering the average dwell-time (17).

For the case of $\mu>1$, under the average dwell-time (17) and the switching condition (S), we can obtain from (40) that

$$
\int_{0}^{t} \mathrm{e}^{-\lambda^{-}(t-\tau)-\lambda \tau} y^{T}(\tau) y(\tau) \mathrm{d} \tau \leq \frac{\gamma_{3}^{2}+\gamma_{d}^{2}}{\gamma_{3}^{2}} \mathrm{e}^{-\lambda^{*} t} V_{\sigma(0)}^{*}(0)+\gamma^{2} \int_{0}^{t} \mathrm{e}^{-\lambda^{*}(t-\tau)} \omega^{T}(\tau) \omega(\tau) \mathrm{d} \tau .
$$

Again, integrating both sides of the foregoing inequality from $t=0$ to $\infty$ and rearranging the double-integral area leads to

$$
\int_{0}^{\infty} \mathrm{e}^{-\lambda \tau} y^{T}(\tau) y(\tau) \mathrm{d} \tau \leq \frac{\lambda^{-}\left(\gamma_{3}^{2}+\gamma_{d}^{2}\right)}{\lambda^{*} \gamma_{3}^{2}} V_{\sigma(0)}^{*}(0)+\gamma_{0}^{2} \int_{0}^{\infty} \omega^{T}(\tau) \omega(\tau) \mathrm{d} \tau
$$

with $\gamma_{0}=\sqrt{\frac{\lambda^{-}}{\lambda^{*}}} \gamma$. From (3), we know that the closed-loop system (1) has the weighted $L_{2}$-gain property.

\section{References}

[1] D. Liberzon, A.S. Morse, Basic problems in stability and design of switched systems, IEEE Trans. Automat. Control 19 (5) (1999) 59-70.

[2] D. Liberzon, Switching in Systems and Control, Birkhäuser, Boston, 2003.

[3] J. Daafouz, P. Riedinger, C. Iung, Stability analysis and control synthesis for switched systems: a switched Lyapunov function approach, IEEE Trans. Automat. Control 47 (11) (2002) 1883-1887.

[4] D. Liberzon, J.P. Hepspanha, A.S. Morse, Stability of switched systems: a Lie-algebraic condition, Systems Control Lett. 37 (3) (1999) $117-122$.

[5] D.Z. Cheng, L. Guo, J. Huang, On quadratic Lyapunov functions, IEEE Trans. Automat. Control 48 (5) (2003) 885-890.

[6] P. Riedinger, M. Sigalotti, J. Daafouz, On the algebraic characterization of invariant sets of switched linear systems, Automatica 46 (6) (2010) 1047-1052.

[7] K.S. Narendra, J. Balakrishnan, A common lyapunov function for stable LTI systems with commuting A-matrices, IEEE Trans. Automat. Control 39 (12) (1994) 2469-2471.

[8] A. Ibeas, M. de la Sen, Exponential stability of simultaneously triangularizable switched systems with explicit calculation of a common Lyapunov function, Appl. Math. Lett. 22 (10) (2009) 1549-1555.

[9] M.S. Branicky, Multiple Lyapunov functions and other analysis tools for switched and hybrid systems, IEEE Trans. Automat. Control 43 (4) (1998) $475-482$.

[10] Z.D. Sun, S.S. Ge, Analysis and synthesis of switched linear control systems, Automatica 41 (2) (2005) 181-195.

[11] F. Long, S.M. Fei, Z.M. Fu, S.Y. Zheng, W. Wei, $H_{\infty}$ control and quadratic stabilization of switched linear systems with linear fractional uncertainties via output feedback, Nonlinear Anal. Hybrid Syst. 2 (1) (2008) 18-27.

[12] J.P. Hespanha, A.S. Morse, Stability of switched systems with average dwell-time, in: Proceedings of the 38th IEEE Conference on Decision and Control, Phoenix, AZ, 1999, pp. 2655-2660.

[13] G.S. Zhai, B. Hu, K. Yasuda, A.N. Michel, Disturbance attenuation properties of time-controlled switched systems, J. Franklin Inst. 338 (7) (2001) 765-779.

[14] J.H. Braslavsky, R.H. Miidleton, Global and semi-global stabilizability in certain cascade nonlinear systems, IEEE Trans. Automat. Control 41 (6) (1996) $876-881$.

[15] A. Chaillet, D. Angeli, Integral input to state stable systems in cascade, Systems Control Lett. 57 (7) (2008) 519-527.

[16] S.D. Huang, M.R. James, Z.P. Jiang, $l_{\infty}$-bounded robust control of nonlinear cascade systems, Systems Control Lett. 54 (3) (2005) $215-224$

[17] J. Mareczek, M. Buss, M.W. Spong, Invariance control for a class of cascade nonlinear systems, IEEE Trans. Automat. Control 47 (4) (2002) 636-640.

[18] W.Z. Su, L.H. Xie, Global robust disturbance attenuation and almost disturbance decoupling for uncertain cascaded nonlinear systems, Automatica 35 (1999) 697-707.

[19] R. Marino, W. Respondek, A.J. van der Schaft, P. Tomei, Nonlinear $H_{\infty}$ almost disturbance decoupling, Systems Control Lett. 23 (3) (1994) 159-168.

[20] L.H. Xie, W.Z. Su, Robust $H_{\infty}$ control for a class of cascaded nonlinear systems, IEEE Trans. Automat. Control 42 (10) (1997) 1465-1469.

[21] A.J. van der Schaft, A state-space approach to nonlinear $H_{\infty}$ control, Systems Control Lett. 16 (1) (1991) 1-8.

[22] M. Wang, J. Zhao, $L_{2}$-gain analysis and control synthesis for a class of uncertain switched nonlinear systems, Acta Automat. Sinica 35 (11) (2009) $1459-1464$

[23] Y.P. Qiao, Design of state-feedback matric in stabilization of block-cascading switched linear systems, in: Proceedings of 2009 IEEE International Conference on Control and Automation, Christchurch, New Zealand, 2009, pp. 220-223.

[24] M. Wang, J. Zhao, Quadratic stabilization of a class of switched nonlinear systems via single Lyapunov function, Nonlinear Anal. Hybrid Syst. 4 (1) (2010) 44-53.

[25] N.N. Song, The single-neural cascade control system of intelligent switch regulating devices and its application, in: Proceedings of the 3rd World Congress on Intelligent Control and Automation, Hefei, PR China, 2000, pp. 1014-1017.

[26] I. Kolmanovsky, N.H. McClamroch, Hybrid feedback laws for a class of cascade nonlinear control systems, IEEE Trans. Automat. Control 41 (9) (1996) 1271-1282. 